\title{
La psicología social en España: estructuras de comunidades
}

\author{
Lupicinio Iñiguez, Juan Muñoz J usticia, \\ María Carmen Peñaranda \& Luz María Martínez \\ Universitat Autònoma de Barcelona ${ }^{1}$
}

\section{Resumen}

Este trabajo se enmarca en el espacio de la 'Psicología social de la ciencia' (Domènech, Íñiguez, Pallí y Tirado 2000; Iñiguez y Pallí, 2002; Moscovici, 1993; Shadish \& Fuller, 1994; Shadish \& Neimeyer, 1989). La Psicología social contribuye a los estudios sociales de la ciencia y la tecnología con el estudio de las interacciones y factores sociales que se dan en el marco de la producción científica y específicamente, promoviendo la idea de que el conocimiento es el resultado de un trabajo conjunto. El presente trabajo considera a la propia Psicología social como objeto de análisis. Específicamente pretende fijar una historiografía de la Psicología social en España, analizar sus características, identificar su dinámica y describir sus prácticas. Para ello, manteniendo las características propias presentes en la corta tradición de la Psicología social de la ciencia, utiliza tanto el Análisis de Redes Sociales (ARS) por su reconocida eficacia en el estudio de comunidades científicas, como la Bibliometría, por su capacidad para analizar la estructura de la producción y la comunicación. En la investigación se analizan las comunicaciones presentadas en los 8 Congresos españoles de Psicología social, celebrados entre 1982 y 2000.

Palabras clave: Psicología Social - Análisis de redes sociales - Bibliometría Comunidades científicas.

\begin{abstract}
This study forms part of a 'social psychology of science" (Domènech, Íñiguez, Pallí y Tirado 2000; Iñiguez y Pallí, 2002; Moscovici, 1993; Shadish \& Fuller, 1994; Shadish \& Neimeyer, 1989). Social psychology's contribution to the social understanding of science and technology is in its study of the interactional and social factors involved in the production of scientific knowledge, exploring the idea that knowledge is the product of joint endeavour. In the work reported here we take Social Psychology itself as the object of study. Specifically, we subject Social Psychology in Spain to a historical analysis, analysing its characteristics, identifying its development and describing its practices. To do so, we follow practices established in the short legacy social psychology of science. We use both social network analysis (SNA), for its acknowledged utility in the study of scientific communities, and bibliometrics, for what it tells us about the structure of scientific publication. We present an analysis of the papers presented at the eight Social Psychology conferences held in Spain between 1982 and 2000.
\end{abstract}

Key words: Social Psychology - Social Network Analysis - Bibliometry - Scientific Comunities.

\footnotetext{
${ }^{1}$ Enviar correspondencia a: Lupicinio Iñiguez Rueda. Lupicinio.iniquez@uab.es
} 


\section{I ntroducción}

Decía Moscovici (1993) que el trabajo científico no es una tarea de individuos aislados, sino que comporta siempre una acción conjunta. La Sociología de la ciencia primero, más tarde la Sociología del conocimiento científico y, más generalmente, los Estudios sociales de la ciencia y de la tecnología, han querido mostrar cómo, mediante qué procesos, con qué determinantes y consecuencias se desarrolla la producción del conocimiento científico y los desarrollos tecnológicos y, además, cómo se produce la comunicación científica y tecnológica y cuál es su impacto social.

En los últimos años la ciencia ha sido objeto de estudio de varias disciplinas de las ciencias humanas y sociales entre las que cabe incluir la Filosofía, la Historia, la Antropología, la Sociología y otras, y la Psicología social no ha sido una excepción. Como consecuencia de abordar la ciencia y la producción científica como objeto de estudio, en la década de los 90 se empiezan a realizar aportaciones que se sitúan a sí mismas en un nuevo espacio denominado "Psicología social de la ciencia" (Moscovici, 1993; Shadish \& Fuller, 1994; Shadish \& Neimeyer, 1989). En otros trabajos anteriores hemos revisado el alcance y los límites del abordaje de la ciencia desde la posición disciplinar de la psicología social (Domènech, 2000; Íñiguez Rueda \& Pallí, 2002).

La propuesta desde la Psicología social es contribuir a los estudios sociales de la ciencia y la tecnología desde el punto de vista que le es históricamente más propio: el estudio de las interacciones y factores sociales que se dan en el marco de la producción científica. Específicamente, promueve la asunción de un enfoque más social donde la producción del conocimiento sea vista como resultado de un trabajo conjunto. Así, la Psicología social de la ciencia no se interesa únicamente por factores sociales ni por factores cognitivos e individuales, sino que investiga la interacción entre ambos así como los procesos mediacionales que permiten pasar de un nivel a otro.

Es en este marco en el que se inserta el presente trabajo. El giro que propone es considerar a la propia Psicología social como objeto de análisis, manteniendo las características propias presentes en la corta tradición de la Psicología social de la ciencia, pero echando mano para ello tanto del Análisis de Redes Sociales (ARS) como de la Bibliometría. El ARS es un área bien establecida en las ciencias sociales que ya ha sido utilizada para el estudio de comunidades científicas (Cronin \& Barsky, 2000; Liberman \& Wolf, 1990; Scott, 2000; Shrum \& Mullins, 1998), mientras que la Bibliometría proporciona un poderoso conjunto de métodos y 
procedimientos técnicos para analizar la estructura de la producción y comunicación científicas (Borgman \& Furner, 2002; Cronin \& Barsky, 2000; Leydesdorff, 2001). Consideramos que éstos son dos enfoques que nos van a permitir fijar una historiografía de la Psicología social en España, analizar sus características, identificar su dinámica y describir sus prácticas.

La Bibliometría consiste en la aplicación de un conjunto de técnicas que buscan cuantificar el proceso de comunicación escrita (Ikpaahindi, 1985). Aunque ha servido principalmente para analizar el volumen de publicaciones, la productividad de autores, revistas o materias, también puede ser utilizada para el conocimiento de los procesos y la naturaleza de las Ciencias, lo que se ha denominado a partir de 1960 Cienciometría o Ciencia de la Ciencia. Existen al menos dos áreas: una descriptiva (tratamiento de aspectos puramente cuantitativos, distribución geográfica, documental, temática y su productividad) y otra evaluativa que añade a la primera estudios de evaluación de la actividad científica.

Es frecuente que las publicaciones (monografías, libros, compilaciones, artículos de revistas, tesis, actas de congresos y reuniones, informes, etcétera) sean la materia base para la evaluación de la actividad científica. Contabilizar la producción, evaluar la actividad investigadora o cartografiar una disciplina, acostumbran a ser los objetivos primordiales de la Bibliometría. Los principales indicadores bibliométricos de la literatura científica son: La productividad de las publicaciones, la productividad de los autores, la productividad por editoriales y lugares de edición, el análisis de la producción por su temática, el análisis de citas y los índices de impacto y el análisis de co-citas y los 'colegios invisibles'.

Así pues, en este trabajo nos valemos de sendas tradiciones, el ARS y la Bibliometría a fin de cartografiar un campo de conocimiento específico, la Psicología social, en un contexto geográfico particular, España.

El propósito de este trabajo es construir un archivo abierto y manejable con la información bibliográfica de los Congresos de Psicología social celebrados en España desde 1983 a 2000 que permita hacer una historiografía de la Psicología social española a través de su análisis. Al hacer esto, creemos estar en condiciones de explorar el alcance y los límites del Análisis de Redes Sociales para el estudio de la actividad científica española en el área de la Psicología social, así como el alcance y los límites del Análisis de Redes Sociales para complementar los estudios de tipo bibliométrico. 
Con este propósito en mente, los objetivos del trabajo son:

- Describir las características básicas de los psicólogos y psicólogas sociales que han participado en los congresos de Psicología social desde 1983 a 2000.

- Describir las características básicas del trabajo de los psicólogos y psicólogas sociales españoles a través del análisis de las comunicaciones presentadas en los congresos de Psicología social.

- Identificar las redes de colaboración de los psicólogos y psicólogas sociales españoles a partir de su participación en los congresos de Psicología social.

- Identificar las comunidades existentes en la Psicología social española por sus afinidades teórico-metodológicas y temáticas.

- Identificar los tópicos principales de la investigación en la Psicología social española.

\section{Construcción del archivo y material de análisis}

Los datos utilizados para el análisis provienen de las comunicaciones publicadas en las actas de los congresos españoles de Psicología social.

Aunque cada vez más los estudios de tipo bibliométrico recurren a bases de datos de referencias como una forma fácil y rápida de obtener los datos fuente, la inexistencia de tal tipo de registro computerizado para el caso de las contribuciones a dichos congresos, nos ha obligado a construir nuestra propia base de datos. En concreto, la base de datos está compuesta por la referencia bibliográfica completa de las comunicaciones, junto con las afiliaciones institucionales de los autores y una clasificación tipológica y la adscripción a un área temática de cada una de las comunicaciones. Igualmente se han incluido todas las referencias bibliográficas citadas en esas comunicaciones. El número total de registros que incluye la base de datos es de 21.482 , de los cuales 1.625 corresponden a comunicaciones y 19.857 a referencias citadas.

A partir de esta base de datos podemos generar las matrices de datos (legibles por los programas de Análisis de Redes) que serán utilizadas para los diferentes análisis. 
La información bibliográfica introducida, fichas bibliográficas de las comunicaciones presentadas en los congresos y de las referencias citadas por dichas comunicaciones (junto con la información de la vinculación entre comunicaciones y referencias), permiten generar diferentes matrices de afiliaciones. Por una parte, la matriz de afiliación autores de comunicaciones x comunicaciones: es decir, dado que cada una de las comunicaciones puede estar firmada por diferentes autores, podemos considerar que los datos origen están representados por una matriz de afiliación o de incidencia en la que las filas (i) serían los 1.499 autores y las columnas $(\mathrm{j}$ ) las 1.625 comunicaciones. Los valores de cada una de las celdas $\mathrm{X}(\mathrm{i}, \mathrm{j})$ pueden ser 1 ó 0 , en función de si la persona i ha participado o no como autor de la comunicación j.

Por otra parte, podemos generar la matriz de afiliación comunicaciones $\mathrm{x}$ referencias citadas, es decir, la información sobre la relación de cada una de las 1.538 comunicaciones (este número es inferior al número total de comunicaciones mencionado anteriormente puesto que no en todos los casos se pudo disponer de información sobre las referencias bibliográficas) con las 20.265 referencias (de nuevo este número es diferente al mencionado anteriormente como total de referencias citadas puesto que 408 comunicaciones son al mismo tiempo referencias citadas por alguna otra comunicación).

Como sabemos, las matrices de afiliación pueden transformarse en matrices de adyacencia a partir de la información de las filas o de las columnas. En el primer caso, la matriz resultante reflejará las relaciones entre los actores en función del número de acontecimientos comunes, mientras que la transformación a partir de las columnas reflejará la relación entre los acontecimientos en función del número de actores comunes. En nuestro caso, transformando la matriz autores $\mathrm{x}$ comunicaciones (a partir de las filas) obtendremos la matriz de coautorías, es decir, la matriz de relaciones entre los autores en función del número de artículos que firman conjuntamente. Por otra parte, para la matriz de comunicaciones $x$ referencias hemos procedido a realizar, en primer lugar, una transformación a partir de las filas, lo que nos da como resultado la matriz de relaciones entre comunicaciones basada en el hecho de compartir referencias, de citar las mismas fuentes; en segundo lugar hemos realizado una transformación en función de las columnas, lo que nos da como resultado la matriz de relaciones entre referencias basada en haber sido citadas por la misma comunicación. 
En la ¡Error! No se encuentra el origen de la referencia. podemos ver una simulación del proceso de transformación.

\begin{tabular}{|c|c|c|c|c|}
\hline & \multicolumn{3}{|c|}{ Comunicaciones } \\
\hline & & $\mathrm{j}_{1}$ & $\mathrm{j}_{2}$ & $\mathrm{j}_{3}$ \\
\hline \multirow{6}{*}{ Autores } & $\mathrm{i}_{1}$ & 1 & & \\
\hline & $\dot{\mathrm{i}}_{2}$ & 1 & 1 & \\
\hline & $\mathrm{i}_{3}$ & 1 & & 1 \\
\hline & $\mathrm{i}_{4}$ & & 1 & 1 \\
\hline & $\mathbf{i}_{5}$ & & & 1 \\
\hline & $i_{6}$ & & & 1 \\
\hline
\end{tabular}

\begin{tabular}{|c|c|c|c|c|c|c|}
\hline & $\mathrm{i}_{1}$ & $\mathrm{i}_{2}$ & $\mathrm{i}_{3}$ & $\mathrm{i}_{4}$ & $\mathrm{i}_{5}$ & $\mathrm{i}_{6}$ \\
\hline $\mathrm{i}_{1}$ & & 1 & 1 & & & \\
\hline $\mathrm{i}_{2}$ & 1 & & 1 & 1 & & \\
\hline $\mathrm{i}_{3}$ & 1 & 1 & & 1 & 1 & 1 \\
\hline $\mathrm{i}_{4}$ & & 1 & 1 & & 1 & 1 \\
\hline $\mathrm{i}_{5}$ & & & 1 & 1 & & 1 \\
\hline $\mathrm{i}_{6}$ & & & 1 & 1 & 1 & \\
\hline
\end{tabular}

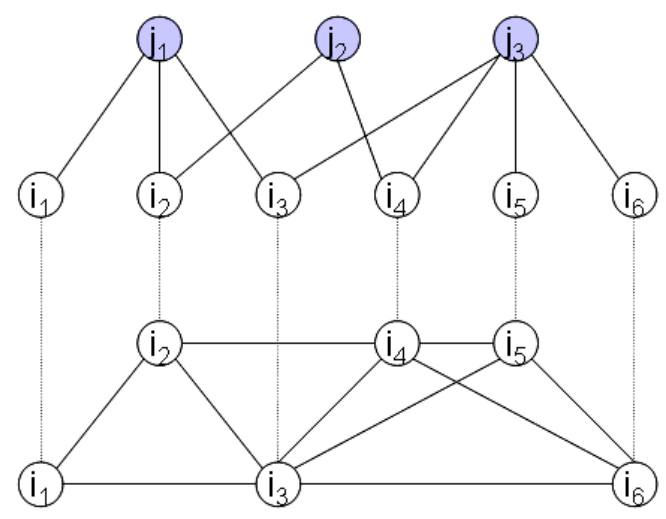

I lustración 1. Conversión de matrices

\section{Análisis y resultados}

\section{Descripción general}

Hemos tomado como corpus de análisis el conjunto de jornadas y congresos de Psicología Social desde el año 1983 hasta el año 2000.

\begin{tabular}{|c|c|c|c|c|c|c|}
\hline CONGRESO & Ciudad & Año & Comunicacione & $\%$ & Autores & $\%$ \\
\hline $\begin{array}{l}\text { III Jornadas de } \\
\text { Psicología Social }\end{array}$ & Las Palmas & 1983 & 27 & 1.67 & 38 & 1,4 \\
\hline $\begin{array}{l}\text { I Congreso de Psicología } \\
\text { Social }\end{array}$ & Granada & 1984 & 113 & 6.96 & 156 & 6,4 \\
\hline $\begin{array}{l}\text { Il Congreso de } \\
\text { Psicología Social }\end{array}$ & Alicante & 1988 & 190 & 11.70 & 259 & 10,8 \\
\hline $\begin{array}{l}\text { III Congreso de } \\
\text { Psicología Social }\end{array}$ & Santiago & 1990 & 183 & 11.27 & 266 & 11,1 \\
\hline $\begin{array}{l}\text { IV Congreso de } \\
\text { Psicología Social }\end{array}$ & Sevilla & 1992 & 327 & 20.13 & 412 & 17,2 \\
\hline $\begin{array}{l}\text { V Congreso de } \\
\text { Psicología Socia }\end{array}$ & Salamanca & 1994 & 246 & 15.14 & 389 & 16,2 \\
\hline $\begin{array}{l}\text { VI Congreso de } \\
\text { Psicología Social }\end{array}$ & Donostia & 1996 & 193 & 11.88 & 312 & 13,0 \\
\hline $\begin{array}{l}\text { VII Congreso de } \\
\text { Psicología Social }\end{array}$ & Oviedo & 2000 & 346 & 21.30 & 557 & 23,3 \\
\hline & & & 1.625 & 100 & $\begin{array}{c}2.38 \\
9\end{array}$ & 100 \\
\hline
\end{tabular}


En el conjunto de congresos (en lo sucesivo no distinguiremos entre Jornadas y Congresos), se han presentado un total de $\mathbf{1 . 6 2 5}$ comunicaciones y ha habido un total de $\mathbf{2 . 3 8 9}$ autores y autoras, de los/as cuales $\mathbf{1 . 4 9 9}$ son distintos. De ellos 601 son mujeres, 592 son hombres y el resto no ha podido ser identificado.

849 autores han presentado una única comunicación, pero hay al menos 1 autor que ha presentado 40. Muchas comunicaciones han sido escritas por autores/as de forma individual (35.33\%), pero la mayor parte de ellas han sido escritas en régimen de co-autoría $(64.67 \%)$. Los datos muestran que el porcentaje de comunicaciones presentadas por un solo autor decrece considerablemente desde el primer congreso $(\mathbf{7 4 . 0 8 \% )}$ hasta el último (30.35\%). Las comunicaciones presentadas en congresos son mayoritariamente colectivas; solamente 154 autores no han contribuido alguna vez con otros, lo que contrasta con 1, que lo ha hecho con 32. En cuanto a la afiliación institucional de los autores, siete universidades contribuyen con más de 100 autores a los congresos de Psicología social. Por orden se trata de las Universidades de Valencia, Autónoma de Barcelona, Sevilla, Barcelona, País Vasco, Complutense y Granada.

\begin{tabular}{|l|r}
\hline Número total de comunicaciones & 1.625 \\
\hline Número total de autores distintos & 1.499 \\
\hline Media de comunicaciones por autor & 2.511 \\
\hline Media de autores por comunicación & 2.316 \\
\hline Colaboradores por autor & 3.833 \\
\hline Tamaño del componente gigante & 754 \\
\hline Tamaño del segundo componente & 95 \\
\hline Distancia media & 8.33 \\
\hline Máxima distancia (diámetro) & 22 \\
\hline
\end{tabular}

Tabla 2. Descripción general

\section{Análisis de la red de coautorías. La Psicología social en España como red}

Este trabajo toma al conjunto de investigadores e investigadoras en Psicología social como red, y se relaciona con aquellos otros que se han centrado específicamente en la comprensión de la estructura de las relaciones sociales analizando redes extensas con el fin de estudiar sus propiedades. M. E. J. Newman (2001) sugirió tomar para este tipo de análisis la comunidad científica: redes de 
científicos y científicas en las que dos de ellos/as se consideran conectados si publican al menos un artículo conjuntamente ${ }^{2}$.

El planteamiento inicial de Newman (2001) introduce ciertas medidas de las redes de colaboración científica como el número de colaboradores que tienen los/as científicos/as, el número de artículos escritos y el grado de "clustering", que es la probabilidad de que dos colaboradores de un científico colaboren ellos mismos. A estas medidas, nosotros hemos introducido algunas otras y ciertos descriptores como la afiliación institucional de los/as autores/as, la tipología de los trabajos, las áreas temáticas en las que se inscriben, así como representaciones gráficas de las redes, muy útiles para analizarlas con precisión.

\section{Descripción de la red de co-autorías. Propiedades básicas de la red}

Las propiedades básicas de la redes sociales tienen consecuencias tanto para las redes tomadas en conjunto como para los/as actores. El grado de integración de una red, así como hasta qué punto los individuos de una red están conectados, son dos aspectos que permiten describir la red desde puntos de vista diferentes. Un asunto central es la composición misma de la red. En efecto, algunas redes están compuestas por actores muy o bastante similares mientras que otras muestran diferencias notables entre ellos. Es el caso de redes donde existe un reducido grupo de nodos centrales y conectados y un amplio número de actores con pocas o ninguna conexión.

En redes muy amplias, como en el caso que vamos a analizar, muchos individuos están, de hecho, aislados. Esto hace muy necesario analizar específicamente las conexiones inmediatas de los actores, o recíprocamente, la distancia entre actores. Estos indicadores ayudan a tener una idea de la difusión, la homogeneidad la cohesión y otras diferencias en las propiedades de las redes y grupos sociales. La distancia y la conectividad son dos de las cuestiones que sería preciso determinar para cada red y el ARS proporciona herramientas específicas para hacerlo.

Como las redes se definen por sus actores y las conexiones existentes entre ellos, se pueden describir el número de actores, el número de conexiones posibles y el número de conexiones efectivamente existentes. Las diferencias en el tamaño y el número de conexiones nos hablan de las diferencias entre grupos grandes y

\footnotetext{
${ }^{2}$ Un planteamiento complementario para el estudio de la red de coautorías en comunidades científicas puede encontrarse en el trabajo de José Luis Molina, Juan Muñoz y Miquel Doménech (2002)
} 
pequeños; por su parte, las diferencias en el número de conexiones nos hablan de la densidad y la "complejidad" de la red. Los actores también difieren entre ellos por el número de conexiones, pueden ser "fuentes" de relaciones o "agujeros" (actores que reciben pero que no emiten). La cantidad de conexiones son informaciones esenciales para saber cuál es su grado de inclusión en una red, cómo ello limita o fortalece su comportamiento así como el rango de oportunidades, influencia o poder que tienen.

Una población puede no estar completamente conectada, es decir, puede que dentro de ella existan dos o más grupos desconectados. Cuando se da el caso de que no todos los actores pueden alcanzar al resto decimos que la población está compuesta por más de un grupo.

El tamaño de la red es crítico para la estructura de las relaciones sociales. A medida que el tamaño crece, la proporción de todos los lazos posibles (densidad) disminuye, apareciendo grupos y facciones diferenciados. En matrices dirigidas, el número de vínculos posibles es ( $*$ * k-1), la mitad cuando la red es simétrica como en nuestro caso. Es decir, para una red como la nuestra compuesta por 1.499 miembros, existen 1.122.751 relaciones lógicamente posibles.

La Media, expresa la densidad de la matriz, es decir, la relación entre el número de conexiones existentes y el número de conexiones posibles. En este caso, sólo el $0.4 \%$ de los lazos posibles están presentes. Por estar próxima a cero, tanto la desviación estándar (0.092) como la varianza (0.008) son muy pequeñas.

Un actor es "accesible" a otro si existe un conjunto de conexiones mediante las cuales se puede trazar un camino, independientemente del número de nodos existentes entre ellos. En datos simétricos como los de nuestra matriz de coautorías, cada par de actores es accesible si uno de ellos está conectado con el otro.

\begin{tabular}{|c|c|c|c|c|c|c|c|c|c|}
\hline & LP & GR & $A L$ & SAN & SEV & SAL & DON & OVI & TOT \\
\hline $\begin{array}{l}\text { DENSIDAD } \\
\text { Desv.est. }\end{array}$ & $\begin{array}{l}0.046 \\
0.208\end{array}$ & $\begin{array}{l}0.016 \\
0.156\end{array}$ & $\begin{array}{l}0.013 \\
0.150\end{array}$ & 0.014 & $\begin{array}{l}0.010 \\
0.137\end{array}$ & $\begin{array}{l}0.007 \\
0.090\end{array}$ & $\begin{array}{l}0.010 \\
0.115\end{array}$ & $\begin{array}{l}0.007 \\
0.104\end{array}$ & $\begin{array}{l}0.004 \\
0.092\end{array}$ \\
\hline & 584 & & .275 & & & & & 75 & 5.481 \\
\hline & & & 7 & & & & & & 7.997 \\
\hline & 552 & 1.596 & 1.275 & 1.444 & 0.999 & 0.656 & 0.973 & 0.733 & 0.366 \\
\hline & & 117 & .460 & .780 & & .514 & & & 0.532 \\
\hline IDAD & $9.46 \%$ & $14.07 \%$ & $9.26 \%$ & $12.99 \%$ & $7.80 \%$ & $1.93 \%$ & $6.14 \%$ & $4.50 \%$ & $6.38 \%$ \\
\hline RING & 1 & 0.879 & 0.898 & 0.882 & 0.816 & 0.849 & 0.886 & 0.852 & 0.810 \\
\hline WEI GHTED & & 0.773 & .648 & 650 & 0.603 & .791 & 0.769 & .812 & .475 \\
\hline
\end{tabular}


Tabla 3. Descriptivos por congreso y total

\section{Componentes de la red}

En redes complejas como la de co-autorías, no siempre cada uno de los/as actores pueden alcanzar a otros. En esos casos podemos decir que existen subpoblaciones separadas. El análisis de redes llama a estas subpoblaciones "componentes".

En la red de co-autorías de los congresos de Psicología social en España existen 35 componentes. Existe además un trigésimo sexto 'componente' constituido por las personas que presentan individualmente sus comunicaciones (en este caso el componente con 391 autores). La mayor parte de los componentes los forman un número muy pequeño de autores. Muy frecuentemente están conformados por grupos de personas que publican una, o muy pocas comunicaciones, siempre firmadas por las mismas o casi las mismas personas. Para nuestra descripción tomaremos en consideración únicamente los componentes grandes: el componente 1, formado por 754 autores; el 3, formado por 95; y el 5, formado por 20 autores y actrices. Estos tres componentes agrupan al $57.97 \%$ de autores si tenemos en cuenta el total de autores, y al $64.61 \%$ si tenemos en cuenta sólo a los autores (1.345) que tienen como mínimo una co-autoría.

Los estadísticos de las matrices de cada componente pueden verse en la Tabla 4.

\begin{tabular}{|l|l|l|l|}
\hline \multicolumn{2}{|c|}{ Componente 1} & Componente 3 & Componente 5 \\
\hline DENSIDAD & 0.010 & 0.065 & 0.253 \\
\hline DESV. ESTÁNDAR & 0.158 & 0.416 & 0.648 \\
\hline CONECTIVIDAD (Degree) & 7.714 & 6.126 & 4.800 \\
\hline Desv. est. & 9.990 & 6.727 & 4.331 \\
\hline CONECTIVIDAD (nrmDeg.) & 1.024 & 6.517 & 25.263 \\
\hline DesV. est. & 1.327 & 7.156 & 22.796 \\
\hline CENTRALIDAD & $12.42 \%$ & $29.20 \%$ & 71.35 \\
\hline CLUSTERING COEFFICIENT & 0.775 & 0.766 & 0.664 \\
\hline WEI GHTED CF & 0.433 & 0.443 & 0.492 \\
\hline
\end{tabular}

Tabla 4. Estadísticos descriptivos componentes

Lógicamente al desglosar la matriz general en sus componentes crece el número de relaciones posibles. Así, para el componente 1 están presentes el $1 \%$ de los vínculos posibles; para el componente 6, el 6.5\%; y para el componente 8 , el $25.3 \%$. La centralidad de la red crece considerablemente en relación a los mismos valores de la población general, siendo la centralidad del $12.42 \%, 29.20 \%$ y $71.35 \%$ respectivamente para los componentes 1,3 y 5 . 


\section{Descripción de la estructura de comunidades}

Algunos estudios recientes de grandes redes se han centrado en el estudio de sus propiedades estadísticas. Michelle Girvan y M. E. J. Newman ("Community structure in social and biological networks," 2001) han estudiado la propiedad de la estructura de comunidades. Se trata de que los nodos de una red estén unidos unos a otros en grupos compactos entre los que sólo hay conexiones débiles.

En efecto, las comunidades podrían ser definidas como subconjuntos de vértices dentro de los cuales las conexiones vértice a vértice son muy densas pero las conexiones entre ellos mucho menos densas. Estos autores han propuesto un algoritmo para identificar estas comunidades basado en la intermediación.

Según Girvan y Newman la detección de comunidades podría tener interesantes aplicaciones prácticas. Por un lado, las comunidades podrían representar agrupaciones sociales reales con intereses o un background común. Por otro, y por ejemplo sería el caso de las redes de citaciones, nos podrían proporcionar agrupaciones de trabajos relacionados con un tema en concreto. En el análisis de páginas Web podrían indicar páginas relacionadas con el mismo tema.

Estos autores proponen un método nuevo para detectar estas agrupaciones que, cuando lo han aplicado a redes bien conocidas y descritas, proporcionan resultados altamente significativos que permiten entender mejor la relación entre la estructura de red y la función.

El procedimiento utilizado se centra en aquellos vínculos que son menos centrales, los vínculos que están más "entre" comunidades. Más que construir agrupaciones por la adición de los vínculos más fuertes con un conjunto de vértices inicialmente vacíos, como haría un análisis de cluster, se construyen los grupos removiendo progresivamente los vínculos del grafo original. Lo que hace en la práctica es identificar los vínculos con mayor intermediación, sacarlos del vector identificando así la estructura de agrupaciones del grafo.

Aplicamos el algoritmo de identificación de comunidades a la red de co-autorías en los congresos de Psicología social a los tres componentes separadamente. El algoritmo proporciona información sobre la agrupación por comunidades a diferentes niveles de agrupación y también información sobre el nivel de significación (best-cut) de cada corte. Para identificar las comunidades seguimos tanto los criterios de significación de cada corte como la interpretabilidad de los resultados. 
Para el caso del componente 1 tomamos el corte 182, con un best-cut 9.660 (significativo 0.001) y que conforma 42 comunidades. Para el caso del componente 3 tomamos el corte 114,5 , con un best-cut 4,552 (significativo 0.001) y que conforma 5 comunidades. En el caso del componente 5 tomamos el corte 22, con un best cut 10,881 (significativo 0,001) y que conforma 2 comunidades. Se han identificado por tanto 49 comunidades. En esta exposición nos centraremos únicamente en las comunidades del componente 1.

Procedemos a una agrupación de los nodos que forman cada una de las 42 comunidades de forma que cada una de ellas pasa a convertirse en un nuevo nodo, con lo que obtenemos una nueva matriz, de $42 \times 42$, en la que cada una de las celdas contiene el número de relaciones (co-autorías) entre los autores miembros de la comunidad i con los autores miembros de la comunidad j. En la Ilustración 1 podemos observar las relaciones entre las diferentes comunidades. El tamaño de los nodos representa el número de miembros de cada una de las comunidades, mientras que el grosor de las líneas representa el número de relaciones entre las comunidades.

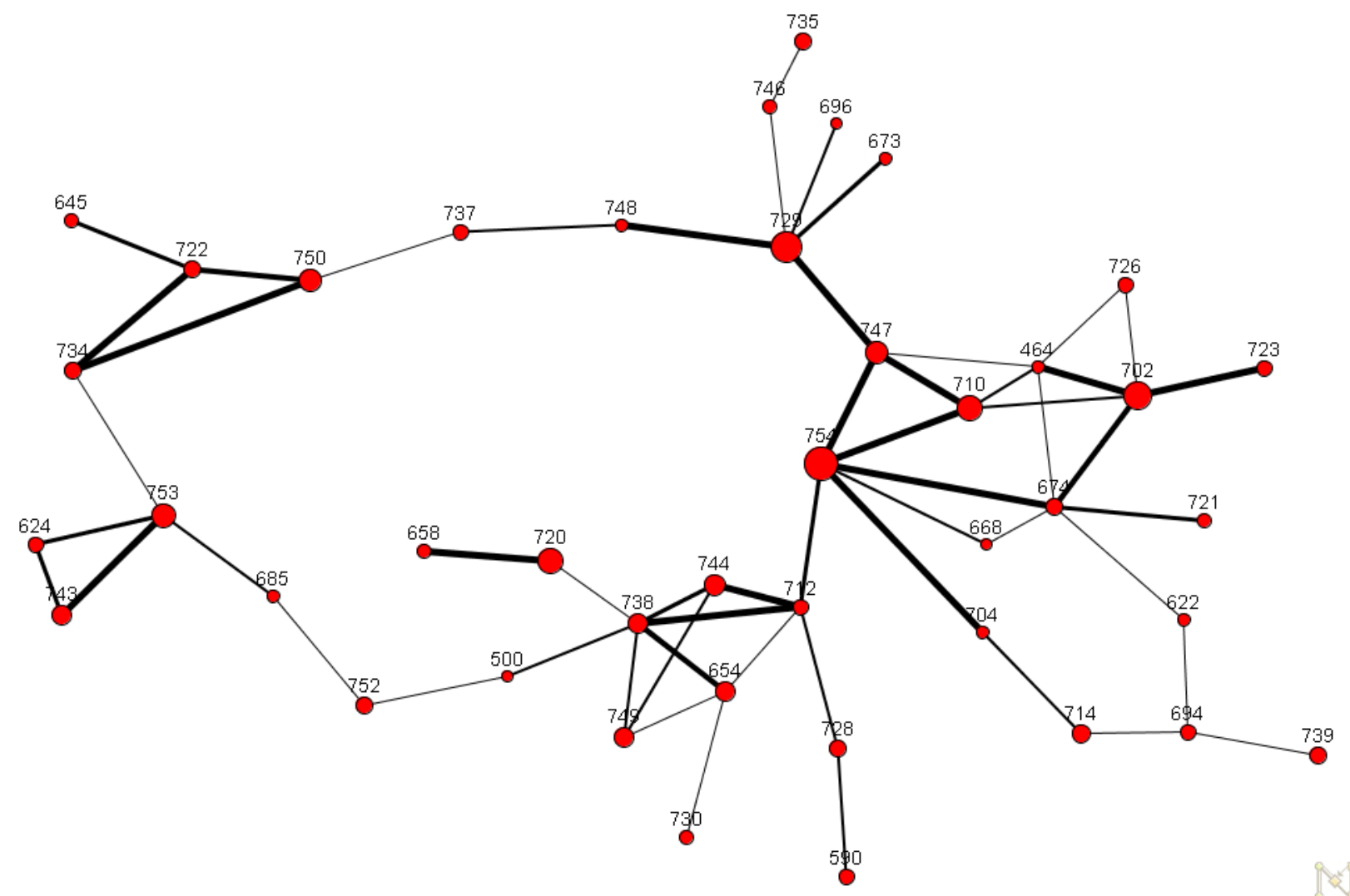

I lustración 1. Relaciones entre comunidades de co-autorías 
Dado que no disponemos de espacio para una descripción completa de la estructura de relaciones entre comunidades, describiremos únicamente seis de ellas, la 624, $743,753,734,722$ y 750 , que se encuentran en la parte izquierda de la llustración 1.

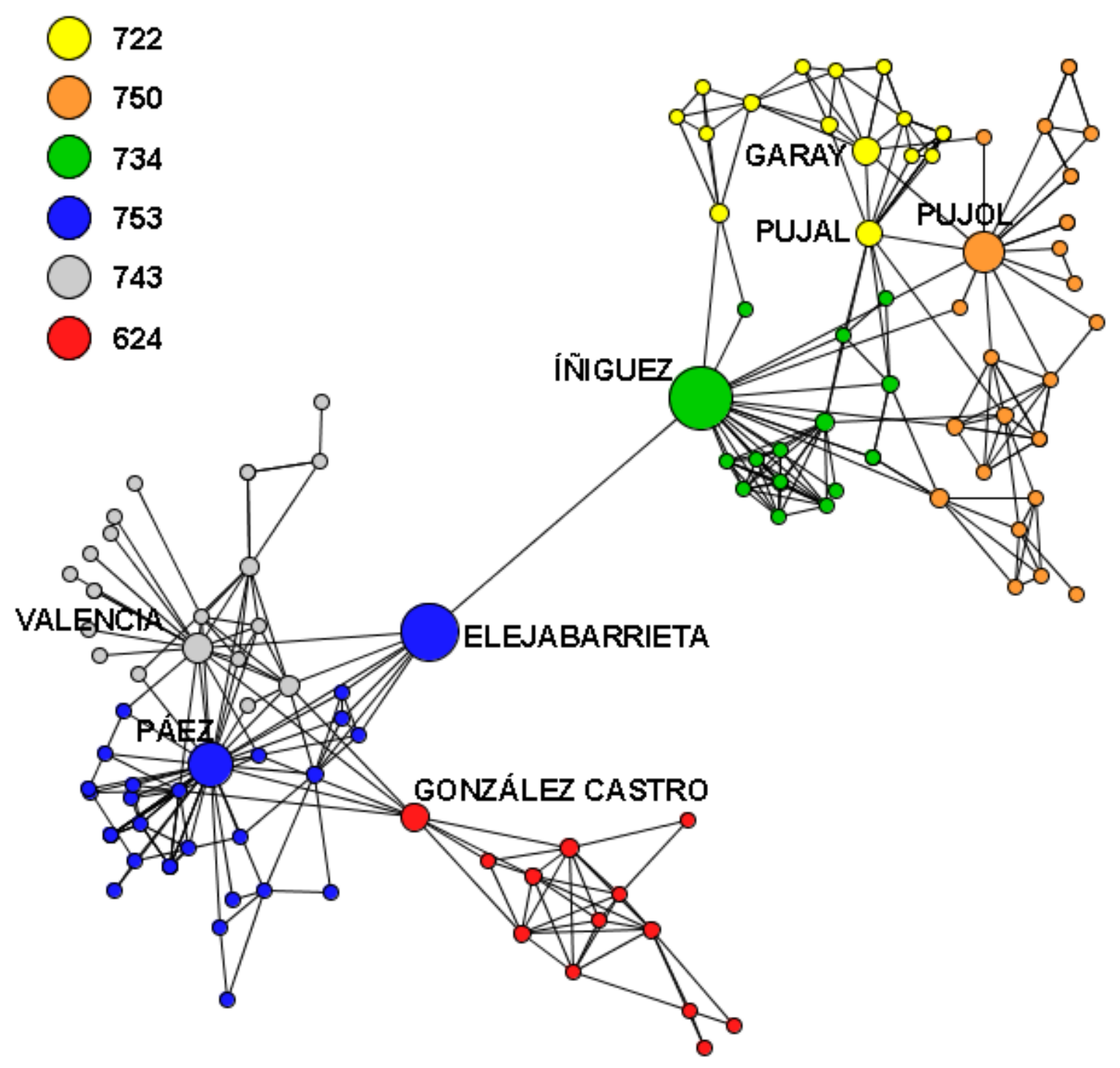

I lustración 2. Selección de comunidades

En la llustración 2 podemos observar las relaciones entre los miembros de las comunidades seleccionadas. En esta ocasión el tamaño de los nodos representa su índice de intermediación. En las siguientes ilustraciones están representados los componentes de cada una de las comunidades. 

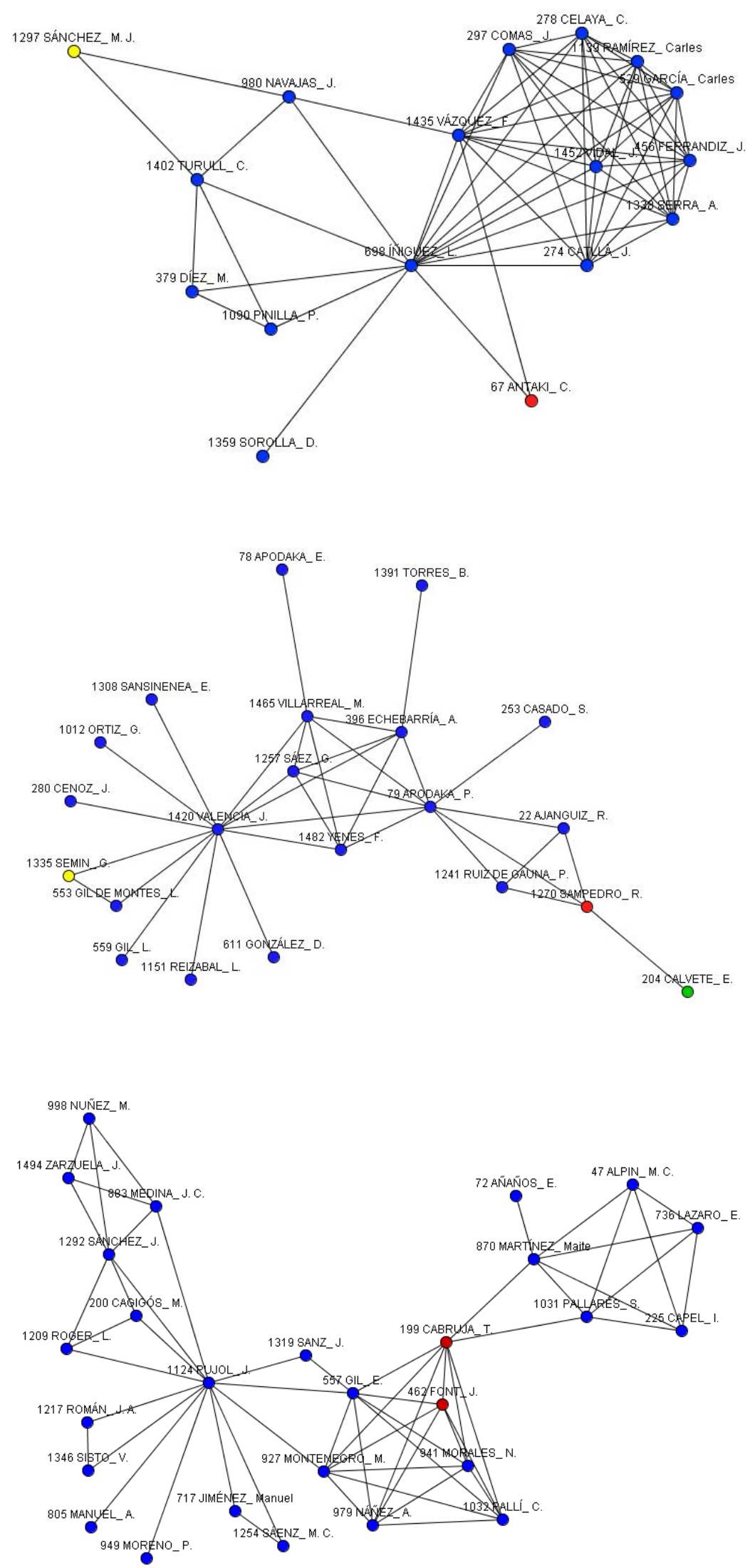


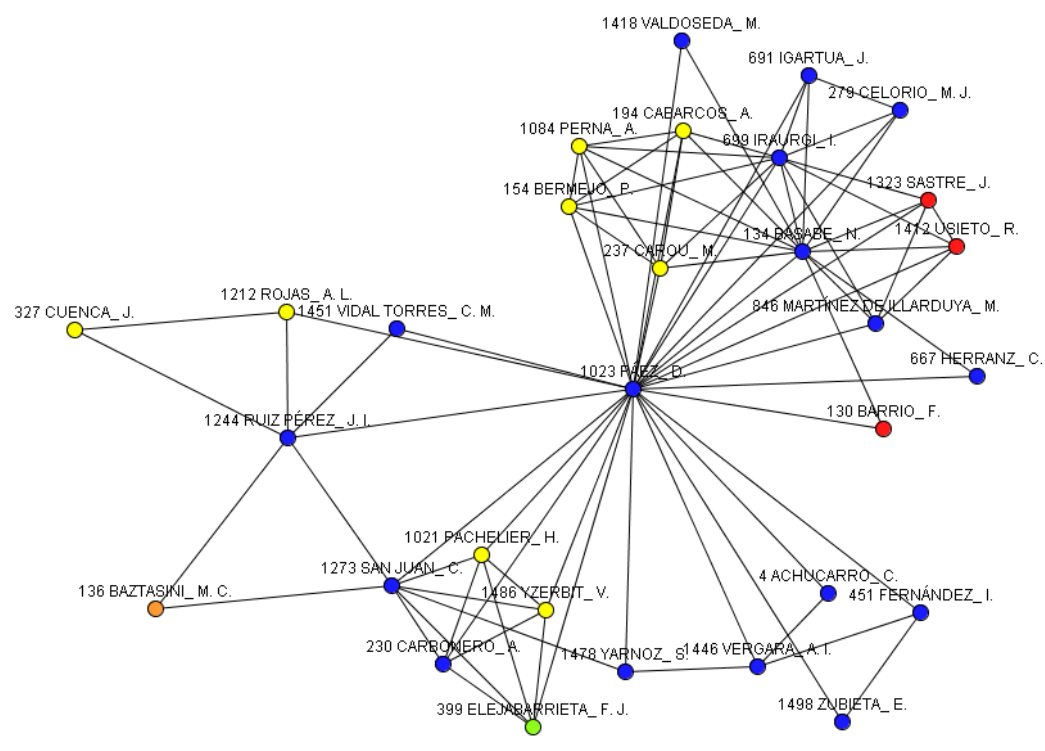

\section{Análisis Bibliométrico}

El análisis de las citas es uno de los indicadores más utilizados en Bibliometría (a partir de los años 60) como medida de la repercusión o impacto de una revista o de un autor. También ha sido utilizado para estudiar el grado de obsolescencia o envejecimiento de la literatura científica. El estudio de las redes de citas ha sido utilizado también para conocer los "colegios invisibles", grupos de profesionales o especialistas que se citan entre sí. La forma habitual de medir hoy día el impacto de las revistas es el cociente de citas que recibe una publicación y las referencias que emite en un período de tiempo (Raising, 1969; Westbrook, 1960).

Es evidente que un trabajo con citas se revaloriza, y que cuando una publicación resulta muy citada implica un impacto. Pero hay elementos que obligan a tratar esta cuestión con cuidado. Parte de estos problemas radica en que la mayor parte de los trabajos no son citados nunca o casi nunca, en el fenómeno de "rebote" que hace que un trabajo citado lo sea cada vez con mayor frecuencia y que un autor puede ser citado "de segunda mano", en los errores técnicos en el análisis de citas derivados de la bases de datos, en la variedad de tipos de citas, etcétera.

Otra forma de enfocar el análisis consiste en explorar la estructura a partir de las co-citas. Las co-citas (dos documentos que son citados conjuntamente por un tercero) pueden analizarse permitiéndonos identificar los "colegios invisibles" (Crane, 1972), es decir, las comunidades informales de personas que trabajan en el mismo tema, que se relacionan de modo diverso entre ellos y que intercambian información. Las posiciones centrales o "cabezas" de un colegio son los autores de 
más alta producción y "visibilidad". Estos autores actúan de receptores y difusores de la información. Las relaciones entre los "miembros" de un "colegio" implican además de una misma línea de investigación, relaciones jerárquicas tanto de tipo institucional como personal o incluso ideológico.

El análisis de co-citas pude ser también un modo de representar la organización intelectual y social de la ciencia, de ofrecer información sobre un campo o especialidad científica o del desarrollo de una disciplina.

En este trabajo presentaremos algunos resultados basados en indicadores de este tipo como el número de citas de los autores y el análisis de co-citas lo que nos permitirá la identificación de grupos de estas características y el mapeo conceptual de la Psicología social.

\section{Análisis de co-citas}

Cuando dos o más trabajos citan una misma referencia, esta coincidencia puede ser interpretada como un indicador de la existencia de un vínculo entre los distintos trabajos. Este criterio se puede endurecer buscando la coincidencia de pares de citas referenciadas simultáneamente por dos o más trabajos. Hablamos en esos casos de co-cita. En este supuesto, el vínculo entre los trabajos es, si se quiere, más intenso y fiable. El análisis de co-citas se basa en estos supuestos y constituye un camino para la identificación de similitudes, ya sean teóricas, metodológicas y/o temáticas entre los trabajos y/o sus autores y las referencias.

A partir de la matriz de afiliaciones de comunicaciones por referencias citadas, hemos elaborado dos matrices de adyacencia, una (a partir de las filas) en la que tendremos las relaciones entre comunicaciones que citan las mismas referencias y otra (a partir de las columnas) en la que se encuentran las relaciones entre referencias que han sido citadas por una misma comunicación.

\section{Aproximación al análisis de los 'colegios invisibles': un enfoque basado en la identificación de comunidades}

Aplicamos nuevamente el algoritmo de identificación de comunidades a la matriz de similitudes entre comunicaciones presentadas en los congresos de Psicología social. En este caso, dada la complejidad del algoritmo, previamente hemos tenido que hacer una reducción de los datos originales, utilizando como criterio para la creación de las matrices la inclusión de únicamente aquellas referencias con una frecuencia de aparición superior o igual a 5, con lo que el tamaño de la matriz de 
relaciones entre comunicaciones será de 556×556. Los nodos son, como sabemos, las comunicaciones que comparten citas entre sí. Los vínculos entre cada par de comunicaciones representan por tanto la coincidencia en al menos un par de citas.

Para identificar las comunidades seguimos tanto los criterios de significación de cada corte como la interpretabilidad de los resultados. Tomamos el corte 832 con un best-cut 35,786 (significativo 0.001) y que conforma 28 comunidades. Estas agrupaciones son indicativas de la estructura de los 'colegios invisibles'. El análisis de las comunicaciones que conforman cada una de las comunidades ha permitido identificarlas provisionalmente del siguiente modo:

\begin{tabular}{|l|r|l|l|}
\hline Etiqueta & $\mathbf{N}$ & Etiqueta & $\mathbf{N}$ \\
\hline $\begin{array}{l}\text { Grupos/Influencia social/Identidad } \\
\text { social }\end{array}$ & 98 & Márquetin social & 4 \\
\hline Procesos sociocognitivos & 68 & Atribución & 4 \\
\hline Educación/Grupos/Organizaciones & 66 & $\begin{array}{l}\text { Organizaciones/Cultura } \\
\text { organizacional }\end{array}$ & 4 \\
\hline PS. Crítica & 60 & Organizaciones/Cambio tecnológico & 3 \\
\hline Salud Comunitaria & 59 & Sexismo & 3 \\
\hline Empleo-Desempleo & 50 & Teoría/Intervención & 3 \\
\hline Conflicto/Negociación & 28 & Comunicación/Narratividad & 3 \\
\hline Organizaciones & 27 & Teoría/Construccionismo & 2 \\
\hline Participación política & 14 & Liderazgo/Influencia & 2 \\
\hline Política/Actitudes valores y creencias & 14 & Drogas/Actitudes y estereotipos & 2 \\
\hline Tiempo libre & 11 & Organizaciones/Liderazgo & 2 \\
\hline Salud/Apoyo social & 8 & Salud/Oncología & 2 \\
\hline Organizaciones/Estrés burnout & 8 & Deporte/Instalaciones & 2 \\
\hline Grupos/Dinámica & 7 & Emociones & 2 \\
\hline
\end{tabular}

Tabla 5. Comunidades de Comunicaciones

De la misma forma que habíamos hecho anteriormente con las comunidades de coautorías, procedemos a una agrupación de los nodos que forman cada una de las 28 , con lo que obtenemos una nueva matriz, de $28 \times 28$, en la que cada una de las celdas contiene el número de relaciones (co-citas) entre los autores miembros de la comunidad i con los autores miembros de la comunidad j. En la Ilustración 3 podemos observar las relaciones entre las diferentes comunidades. Como podemos apreciar, del total de 28 comunidades, existen relaciones entre 16 de ellas, mientras que las otras 12, que no se incluyen en la ilustración, no están relacionadas con ninguna otra. El tamaño de los nodos representa el número de miembros de cada una de las comunidades, mientras que el grosor de las líneas representa el número de relaciones entre las comunidades. 


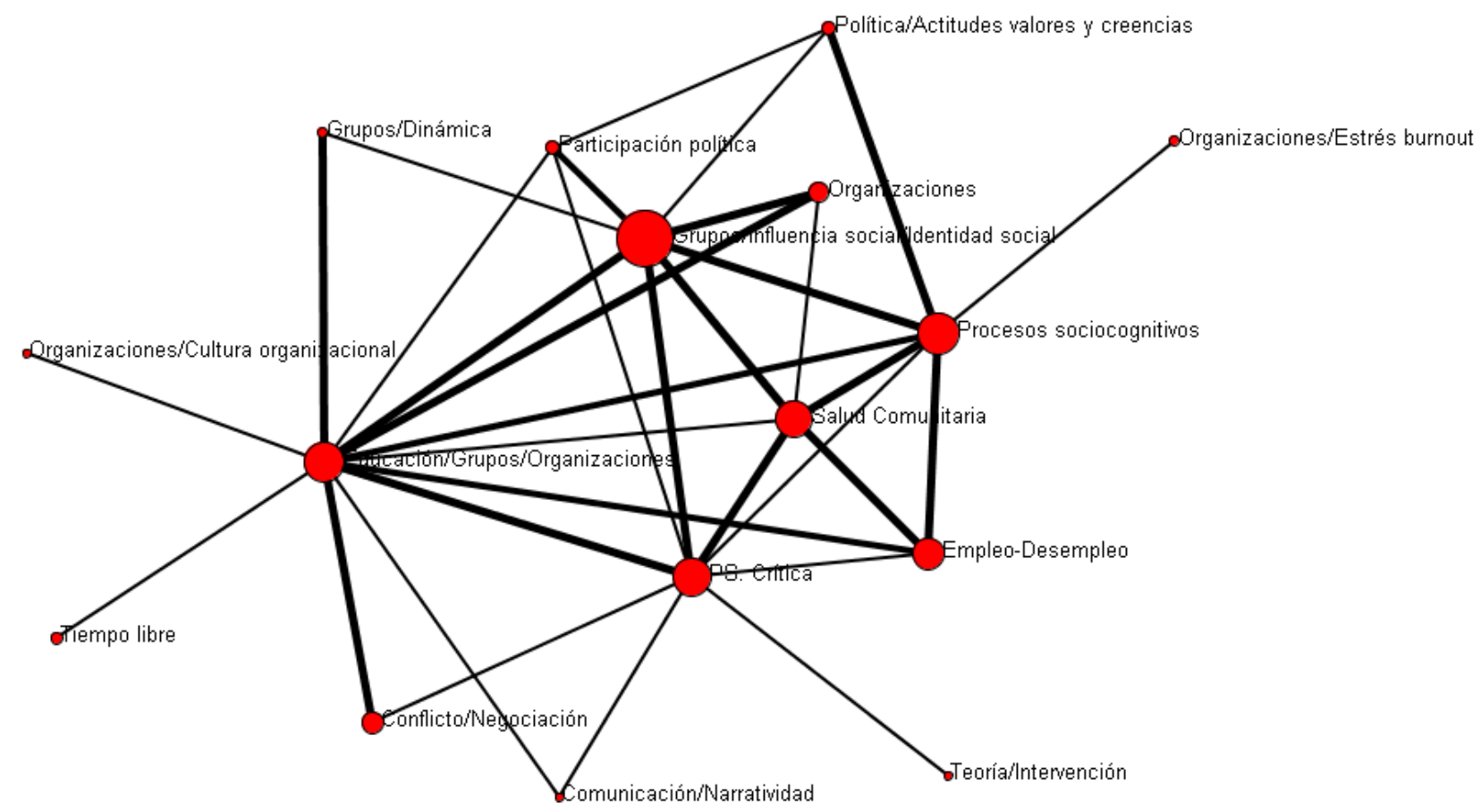

I lustración 3. Relaciones entre comunidades de co-citas

\section{Aproximación al mapa teórico-metodológico y temático: un enfoque basado en la identificación de comunidades}

Aplicamos también el algoritmo de identificación de comunidades a la matriz de similitudes entre referencias citadas en las comunicaciones presentadas en los congresos de Psicología social. Los 453 nodos $^{3}$ son, como sabemos, las referencias que aparecen en pares de co-citas. Los vínculos entre cada par de referencias representan por tanto la coincidencia en al menos un par de comunicaciones.

Para identificar las comunidades seguimos tanto los criterios de significación de cada corte como la interpretabilidad de los resultados. El algoritmo aplicado a esta matriz produce los resultados siguientes. Tomamos el corte 108 con un best-cut 18,458 (significativo 0.001) y que conforma 53 comunidades pero de las que 27 están compuestas de una única referencia. Consideramos por tanto las 26 restantes. Las hemos identificado provisionalmente del siguiente modo:

\begin{tabular}{|l|l|l|l|}
\hline Etiqueta & $\mathbf{N}$ & Etiqueta & $\mathbf{N}$ \\
\hline
\end{tabular}

\footnotetext{
${ }^{3}$ Como hemos comentado anteriormente, se procedió a una reducción de la matriz original seleccionando únicamente aquellas referencias que aparecían citadas un mínimo de cinco ocasiones.
} 


\begin{tabular}{|l|r|l|r|}
\hline Transversal & 93 & Individualismo-Colectivismo & 7 \\
\hline P.S. Crítica & 50 & Ocio y tiempo libre & 7 \\
\hline Apoyo social & 39 & Ambiental & 6 \\
\hline Empleo/Desempleo & 28 & Organizaciones/Liderazgo & 5 \\
\hline Comunitaria/Salud & 25 & P.S./Cambio de actitud & 4 \\
\hline Actitudes valores y creencias & 25 & Educación/Motivación & 3 \\
\hline Grupos/Organizaciones & 25 & Representaciones sociales & 3 \\
\hline Organizaciones/Psicometría & 21 & Márquetin social & 3 \\
\hline Participación política & 20 & Sexo-Género & 2 \\
\hline Conflicto/Negociación & 19 & Salud/Oncología & 2 \\
\hline Influencia social & 14 & Sexo-Género/Sexismo & 2 \\
\hline Lenguaje/Identidad Social & 11 & Deporte & 2 \\
\hline Organizaciones/Estrés-Burnout & 8 & Empleo- & 2 \\
\hline & & & \\
\hline
\end{tabular}

Tabla 6. Comunidades de Referencias

Estas agrupaciones representan agrupaciones teórico-temáticas que permitirán, eventualmente cartografiar la Psicología social española.

En la llustración 4 podemos ver la estructura de relaciones entre las comunidades.

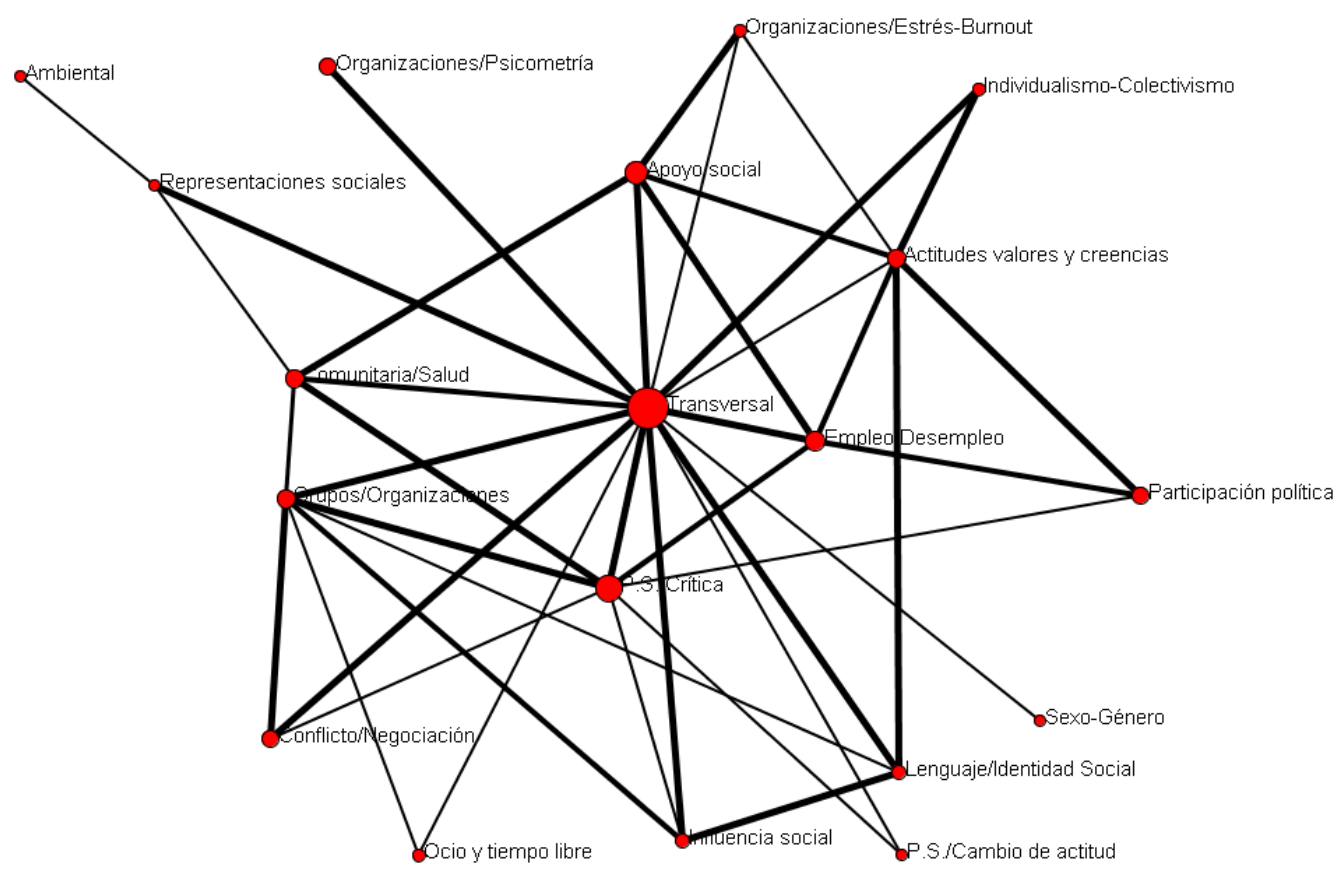

I lustración 4. Relaciones entre comunidades de co-citas (referencias)

\section{Discusión y prospectiva}

Los congresos y reuniones científicas son una parte integral de la actividad científica. Sin embargo, han sido frecuentemente ignorados como un tópico independiente de investigación en los estudios sociales de la ciencia y la tecnología. 
No ha sido así en el caso de las publicaciones que se han constituido en elementos centrales para el estudio de la práctica y los desarrollos científicos y tecnológicos.

Sin embargo, los congresos y reuniones científicas son una parte esencial de la práctica científica, tanto como vehículo de información y diseminación de teorías y análisis empíricos, como contexto de establecimiento de vínculos y relaciones (Martens \& Saretzki, 1994; Söderqvist \& Silverstein,1994a, 1994b). Las reuniones científicas son "arenas' de conocimiento especializado donde se establecen nuevos vínculos a través de la relación y del intercambio de información. Las informaciones fluyen a través de los contactos que se establecen en las reuniones. Son también contextos especialmente importantes en el inicio de la carrera de jóvenes investigadores e investigadoras constituyendo uno de los ámbitos más importantes para la socialización académica. Entendemos que los estudios sociales de la ciencia no deberían desatender por más tiempo esta parte tan importante de la práctica científica, si lo que se pretende es conocerla.

Así pues, las reuniones científicas merecerían la atención de los estudios de la ciencia por múltiples razones, aunque sólo sea para comprender el establecimiento y la dinámica de disciplinas científicas y áreas de investigación, su evolución y transformaciones, y la constitución de sus áreas y grupos de trabajo.

En este sentido, algunas preguntas podrían ser respondidas con relativa facilidad analizando los congresos y otros tipos de reuniones científicas. En el caso de la Psicología social:

- ¿Podemos identificar a los "líderes" de la Psicología social y sus grupos?

- ¿Cuáles son sus subdisciplinas, sus áreas de investigación, sus tópicos de interés, etcétera?

- ¿Cómo se relacionan estas subdisciplinas, áreas tópicos y/o orientaciones las unas con otras?

- ¿Se puede cartografiar la dinámica disciplinaria de la Psicología social? ¿Podemos determinar cómo y cuándo áreas de investigación periféricas con sus propios programas de investigación se unen al mainstream de la Psicología social?

En este trabajo preliminar sólo hay una indicación de que estas preguntas pueden ser respondidas y se ofrece únicamente un camino que permitiría llevar a esos resultados. 
Hemos elegido la propia Psicología social para este intento y, específicamente sus congresos realizados en España. La principal idea que subyace a nuestro análisis es que un análisis de la participación en reuniones basado en el ARS y la bibliometría puede proporcionar información sobre la estructura y la dinámica de la comunidad de la Psicología social durante el periodo de su progresiva disciplinarización.

El "quién es quién" de la Psicología social española resulta claramente identificable cuando se analizan las contribuciones a los congresos y el conjunto de la literatura referenciada.

El uso del algoritmo de identificación de comunidades nos abre el camino para distinguir los grupos constitutivos de la Psicología social española, así como sus áreas y tópicos de interés. Éste es un procedimiento novedoso que viene a complementar los enfoques anteriores en la identificación de los 'colegios invisibles'.

Las áreas y sub-áreas, así como los tópicos de interés de la Psicología social española resultan igualmente identificables utilizando el mismo procedimiento. En este punto de la investigación, aún por concluir, nos hemos limitado a ejemplificar su alcance con la descripción de sólo una parte de ellos, pero creemos haber establecido sobradamente que es un camino interesante a seguir. Las ventajas de este procedimiento respecto a los más habituales, como el análisis de cluster, resultan claras y esperanzadoras.

\section{Bibliografía}

Borgman, Christine L. \& Furner, Jonathan (2002). "Scholarly Communication and Bibliometrics". en B. Cronin (Ed.), Annual Review of Information Science and Technology Medford, NJ .: Information Today Inc., (pp. 3-72).

Crane, Diana (1972). Invisible colleges. Diffusion of knowledge in scientific communities. Chicago: University of Chicago Press.

Cronin, Blaise \& Barsky, Helen (2000). The web of knowledge. A festchrift in honor of Eugene Garfiel. Medford, NJ .: Information Today.

Domènech Argemí, Miquel, Íñiguez Rueda, Lupicinio, Pallí, Cristina \& Tirado Serrano, Francisco Javier (2000). "La contribución de la psicología social al estudio de la ciencia". Anuario de Psicología, Vol. 31, № 3, 77-93.

Girvan, M. \& Newman, M. E. (2001). Community structure in social and biological networks. http://arxiv.org/PS_cache/cond-mat/pdf/0112/0112110.pdf .

I kpaahindi, Linus (1985). "An overview of bibliometrics: its measurements, laws, and their applications". Libri, Vol. 35, № 2, 163-177. 
Íñiguez Rueda, Lupicinio \& Pallí, Cristina (2002). "La Psicología social de la ciencia: Revisión y discusión de una nueva área de investigación". Anales de Psicología, no 18, 13-43.

Leydesdorff, Loet (2001). The challenge of scientometrics. The development, measurement and self-organization of scientific communication. 2 Parkland, FL.: Universal Publishers.

Liberman, Sofía \& Wolf, Kurt Bernardo (1990). Las redes de comunicación científica. Cuernavaca: Universidad Nacional Autónoma de México.

Martens, B.\& Saretzki, T. (1994). "Quantitative analysis of thematic structures in the field of biotechnology: A study on the basis of conference data". Scientometrics, Vol. 30, no1, 117-128.

Milgram, Stanley (1967). "The small world problem". Psychology Today, Vol. $\underline{67}$, no $1,60-67$.

Molina, José Luis, Muñoz, Juan y Domènech, Miquel (2003). Redes de publicaciones científicas: un análisis de la estructura de coautorías". Redes. Revista hispana para el análisis de redes sociales, 1, Artículo 3. Consulta [15-06-2006] en http://revistaredes.rediris.es

Moscovici, Serge (1993). "Toward a Social Psychology of Science". Journal for the Theory of Social Behaviour, Vol. 23, no 4, 343-374.

Newman, Mark E.J. (2001). "The structure of scientific collaborative networks". Proceedings of the National Academy of Sciences JO - PNAS, Vol. 98, no 2, 404409.

Raising, L.M. (1969). "Mathematical evaluation of the Scientific serial". Science, Vol. 131, 1417-1419.

Requena Santos, Félix (1998). "Redes (sociales), análisis de". en S. Giner, E. Lamo de Espinosa et al. (Eds.) Diccionario de Sociología Madrid: Alianza Editorial,

Scott, J ohn (2000). Social network analysis. A handbook. 2 London: Sage. Shadish, William R. \& Fuller, Steve The Social Psychology of Science. .

Shadish, William R. \& Neimeyer, Robert A. (1989). "Contributions of psychology to an integrative science studies: The shape of things to come". en S. Fuller, M. De Mey et al. (Eds.) The cognitive turn: Sociological and psychological perspectives on science Dordrecht: Dluwer, (pp. 13-38).

Shrum, Wesley \& Mullins, Nicholas (1998). "Network analysis in the study of science and technology". en A. van Raan (Ed.), Handbook of Quantitative Studies in Science and Technology Amsterdam: Elsevier Science Publishers, (pp. 107-133.

Söderqvist, Thomas \& Silverstein, Arthur M. (1994a). “Studying leadership and subdisciplinary structure of scientific disciplines. Cluster analysis of participation in Scientific Meetings". Scientometrics, Vol. 30, no 1,243-258

Söderqvist, Thomas \& Silverstein, Arthur M. (1994b). "Participarion in Scientific Meetings: A New Prosopographical Approach to the Disciplinary History of Science The Case of Immunology, 1951-72". Social Studies of Science, Vol. 24, 513-548 
Westbrook, J. H. (1960). "I dentifying significant research". Science, Vol. 132, 1229-1234. 